\title{
Immunologic Monitoring of Cellular Immune Responses in Cancer Vaccine Therapy
}

\author{
Theresa L. Whiteside, ${ }^{1}$ James L. Gulley, ${ }^{2}$ Timothy M. Clay, ${ }^{3}$ and Kwong Yok Tsang ${ }^{2}$ \\ ${ }^{1}$ Department of Pathology, University of Pittsburgh School of Medicine and University of Pittsburgh Cancer Institute, Pittsburgh, \\ PA 15213-1863, USA \\ ${ }^{2}$ Laboratory of Tumor Immunology and Biology, Center for Cancer Research, National Cancer Institute, National Institutes of Health, \\ Bethesda, MD 20892, USA \\ ${ }^{3}$ Department of Surgery, Duke University Medical Center, Durham, NC 27710, USA
}

Correspondence should be addressed to Kwong Yok Tsang, tsangkwo@mail.nih.gov

Received 7 April 2011; Accepted 7 April 2011

Copyright (c) 2011 Theresa L. Whiteside et al. This is an open access article distributed under the Creative Commons Attribution License, which permits unrestricted use, distribution, and reproduction in any medium, provided the original work is properly cited.

Immunotherapy for cancer is based on the concept of inducing the generation and expansion of immune cells that can attack and eliminate cancer. Immunotherapy with therapeutic cancer vaccines aims specifically at inducing tumor antigen-specific $\mathrm{T}$ cells. The immune system is a complex, multifaceted cellular network that is not fully understood. Monitoring cellular immune responses is essential for rational cancer vaccine development. The primary objectives of immune monitoring after vaccination are to document the induction of vaccine-specific and tumorspecific immune responses and to correlate the presence and magnitude of vaccine-induced immune responses to clinical outcomes. Immune monitoring could also be used to (a) define the ability of a given vaccine to generate antigen cascade responses (i.e., epitope spreading); (b) compare the effects of vaccines of different potencies; (c) evaluate the ability of a given cytokine, drug, adjuvant, and so forth. to enhance or hinder vaccine-induced immune responses; (d) define appropriate patient populations for vaccine studies; (e) study the presence and activity of inhibitory/ suppressor cell populations.

Despite advances in the development of immune monitoring assays during the past decade, it has been difficult to establish significant correlations between vaccine-induced immune responses and clinical outcomes. This lack of correlation could reflect the methodological limitations of immunologic assays or the postvaccination absence of antitumor responses sufficiently robust to induce diseasefree or overall survival. A wide portfolio of monitoring assays is currently available. However, these assays fail to define surrogate markers that could be used as predictors of clinical response and thus serve to advance vaccine development. The immune monitoring assays currently used in cancer immunotherapy trials (such as enzyme-linked immunospot assays, tetramer-based assays, intracellular cytokine flow cytometry, antibody tests, proliferation assays, reverse transcription polymerase chain reaction, and serum cytokine and chemokine profiles) have limited usefulness as surrogate markers of clinical efficacy. There is general consensus that further studies are needed to account for the difficulties in establishing the correlation between different aspects of T-cell function and clinical efficacy.

Vaccine-induced immune responses against cancer depend on a balance between immune responses of various subsets of effector and suppressor $\mathrm{T}$ cells. Because tumor antigens are mostly self-antigens, this balance is shifted toward tolerance in cancer patients, so that generating effective antitumor responses requires breaking of tolerance. Although preclinical data have shown that it is possible to break tolerance to tumor-associated selfantigens, human clinical trials employing cancer vaccines have mostly failed to do so. In an immunocompetent cancer patient, the immune system suppresses attacks against selfantigens, including tumor-associated antigens, particularly in the tumor microenvironment. Recent studies have focused on defining the role of the suppressive component of the antitumor immune response in breaking tolerance and steering the immune system toward autoimmunity. In this 
respect, monitoring assays that measure the extent of cancerinduced suppression may be especially important.

The suppressive compartment of the immune system includes a group of heterogeneous immune cells, including regulatory $\mathrm{T}$ cells (Tregs), myeloid-derived suppressor cells (MDSCs), and tumor-associated macrophages (TAMs). Increased numbers and/or enhanced functionality of these cells have been detected in the peripheral blood mononuclear cells, tumor microenvironment, and tumor-draining lymph nodes of patients with hematologic malignancies and various types of solid tumors. One of the major problems in characterizing these cells is their extreme plasticity. Cells normally committed to activating an immune response can transiently acquire suppressive characteristics, as often happens in cancer. While suppressor cells represent an important mechanism by which the immune system finetunes specific immune responses, expansion of these cells in cancer patients interferes with antitumor immunity. In humans, it has been difficult to establish a definite phenotype for these cells, and assessment of their functional status has been a special challenge as they are minor lymphocyte subsets lacking well-defined surface markers. Greater understanding of the mechanisms that regulate the homeostasis of these suppressive cells could lead to the development of more effective cancer immunotherapies and better immune monitoring of patients receiving cancer vaccines.

Several studies have demonstrated that Treg depletion can efficiently enhance vaccine-mediated antitumor immunity in cancer patients. For example, in a randomized placebo-controlled multicenter phase II trial, 125 patients with metastatic castration-resistant prostate cancer were treated with a poxviral-based vaccine containing the transgenes for prostate-specific antigen and 3 costimulatory molecules (PSA-TRICOM). This trial demonstrated a direct correlation between the post-vaccination frequency and function of Tregs and overall survival. Treg function and/or phenotype, as well as the ratio of effector to CTLA- $4^{+}$ Tregs, could potentially be used to monitor immune function (the balance between immunostimulatory and immunosuppressive factors) in patients enrolled in clinical trials of therapeutic cancer vaccines $[1,2]$. Analysis of Tregs in real time as part of the immune monitoring of patients could also help to identify the subpopulation of patients who would most likely benefit from vaccine therapy.

Similar to Tregs, MDSCs are a heterogeneous cell population that has been difficult to monitor in humans. MDSCs are composed mainly of myeloid progenitor cells that do not completely differentiate into mature macrophages, dendritic cells, or granulocytes. A recent study demonstrated that a subpopulation of monocytic MDSCs, phenotypically defined as CD $14^{+}$HLA-DR ${ }^{-/ l o}$, is significantly expanded in patients with metastatic melanoma, hepatocellular carcinoma, glioblastoma, and prostate cancer. Increased circulating MDSCs have also been correlated with tumor stage and metastatic spread in different types of tumors [3, 4]. Moreover, it has been demonstrated that MDSCs can be differently affected by standard-of-care therapies such as sunitinib, doxorubicin-cyclophosphamide, and docetaxel, as well as some immunotherapies. These findings suggest a potential use for these cells in immune monitoring of cancer patients receiving immunotherapies.

Recent clinical studies have demonstrated a correlation between increased numbers of TAMs and poor prognosis for esophageal, bladder, prostate, endometrial, breast, and lung cancers [5-8]. These data suggest a possible use of TAMs in the immune monitoring of cancer patients enrolled in clinical trials employing therapeutic vaccines.

While the major focus of post-vaccination monitoring is assessment of tumor antigen-specific immune responses, it is important to note that innate immunity mediated by natural killer cells, macrophages, dendritic cells, or granulocytes could significantly contribute to beneficial clinical outcome. Vaccines that engage and promote adaptive and innate antitumor responses appear to be most effective. Thus, at least some aspects of innate immunity should be monitored in clinical trials of antitumor vaccines.

The increasing number of immunotherapy clinical trials that use immunologic parameters as primary or secondary endpoints, as well as the availability of an increasing number of monitoring assays, highlights two aspects of immune monitoring. First, selection of assays that are most likely to correlate with clinical outcomes is a critical factor. This should be an informed and hypothesis-driven choice, considering factors such as cost and the need for serial monitoring with multiple assays. As understanding of the mechanisms of immune regulation increases, additional biomarkers will be identified that, hopefully, can be used as surrogate markers for immune responses to cancer vaccines. Second, quality control and assurance are essential for successful immune monitoring of cancer vaccines. All immune monitoring assays need to be standardized for reliability and consistency in order to establish their limitations and overall performance standards. Immune monitoring assays should be performed according to Good Laboratory Practice guidelines, such as those recently formulated based on recommendations from the iSBTc-SITC/FDA/NCI Workshop on Immunotherapy Biomarkers [9]. Only standardized monitoring assays are likely to be useful for defining surrogate endpoints of clinical response to antitumor vaccines.

\section{Theresa L. Whiteside James L. Gulley Timothy M. Clay Kwong Yok Tsang}

\section{References}

[1] M. Vergati, V. Cereda, R. A. Madan et al., "Analysis of circulating regulatory $\mathrm{T}$ cells in patients with metastatic prostate cancer pre- versus post-vaccination," Cancer Immunology, Immunotherapy, vol. 60, no. 2, pp. 197-206, 2011.

[2] J. L. Gulley, P. M. Arlen, R. A. Madan et al., "Immunologic and prognostic factors associated with overall survival employing a poxviral-based PSA vaccine in metastatic castrate-resistant prostate cancer," Cancer Immunology, Immunotherapy, vol. 59, no. 5, pp. 663-674, 2010.

[3] P. Filipazzi, R. Valenti, V. Huber et al., "Identification of a new subset of myeloid suppressor cells in peripheral blood 
of melanoma patients with modulation by a granulocytemacrophage colony-stimulation factor-based antitumor vaccine," Journal of Clinical Oncology, vol. 25, no. 18, pp. 25462553, 2007.

[4] S. Vuk-Pavlović, P. A. Bulur, YI. Lin et al., "Immunosuppressive CD14+HLA-DR monocytes in prostate cancer," Prostate, vol. 70, no. 4, pp. 443-455, 2010.

[5] N. Koide, A. Nishio, T. Sato, A. Sugiyama, and S. I. Miyagawa, "Significance of macrophage chemoattractant protein-1 expression and macrophage infiltration in squamous cell carcinoma of the esophagus," American Journal of Gastroenterology, vol. 99, no. 9, pp. 1667-1674, 2004.

[6] T. Hanada, M. Nakagawa, A. Emoto, T. Nomura, N. Nasu, and Y. Nomura, "Prognostic value of tumor-associated macrophage count in human bladder cancer," International Journal of Urology, vol. 7, no. 7, pp. 263-269, 2000.

[7] S. Ohno, Y. Ohno, N. Suzuki et al., "Correlation of histological localization of tumor-associated macrophages with clinicopathological features in endometrial cancer," Anticancer Research, vol. 24, no. 5, pp. 3335-3342, 2004.

[8] B. C. Zhang, J. Gao, J. Wang, Z. G. Rao, B. C. Wang, and J. F. Gao, "Tumor-associated macrophages infiltration is associated with peritumoral lymphangiogenesis and poor prognosis in lung adenocarcinoma," Medical Oncology.

[9] L. Butterfield, A. Palucka, C. Britten et al., "Recommendations from the iSBTc-SITC/FDA/NCI workshop on immunotherapy biomarkers," Clinical Cancer Research, vol. 17, no. 10, pp. 30643076, 2011. 


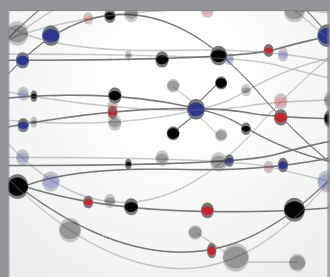

The Scientific World Journal
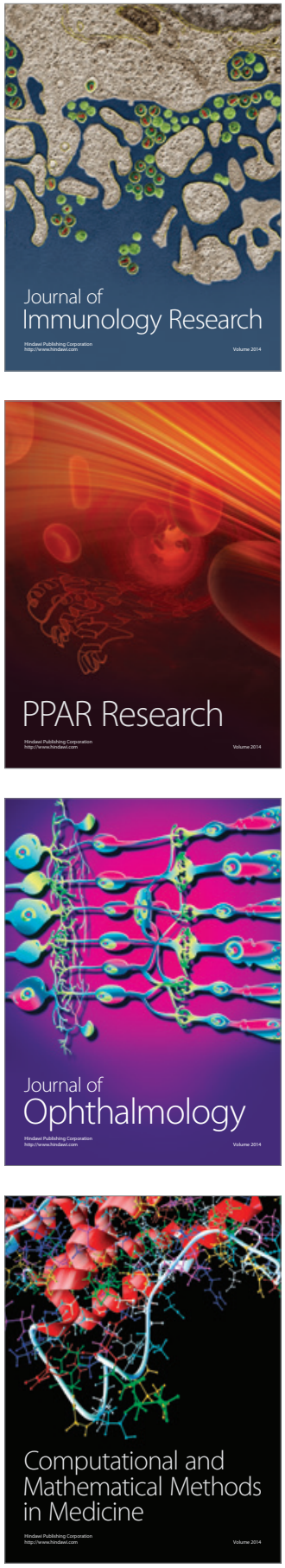

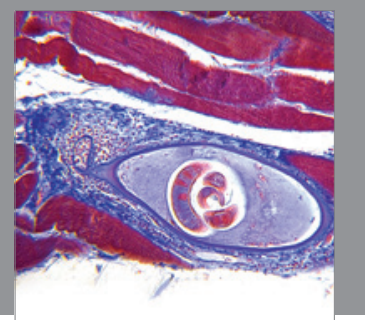

Gastroenterology

Research and Practice
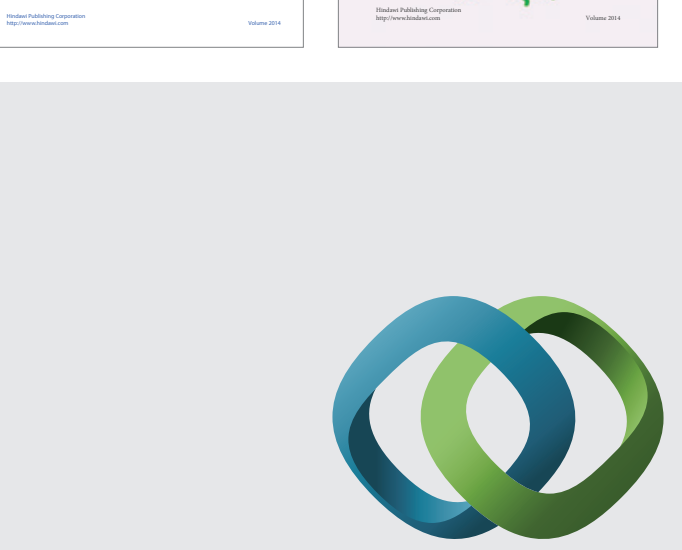

\section{Hindawi}

Submit your manuscripts at

http://www.hindawi.com
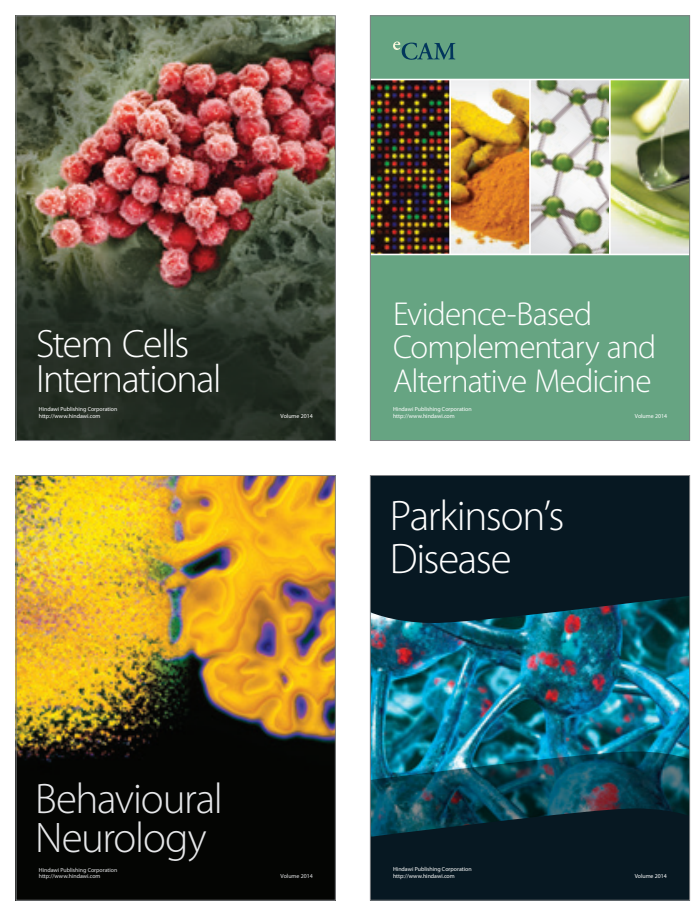

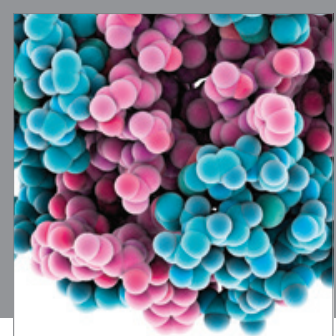

Journal of
Diabetes Research

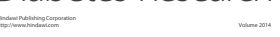

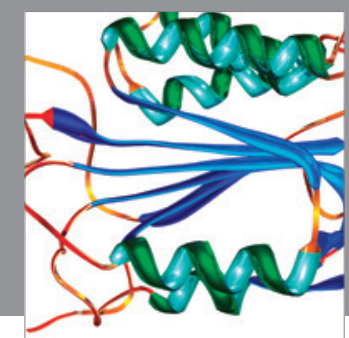

Disease Markers
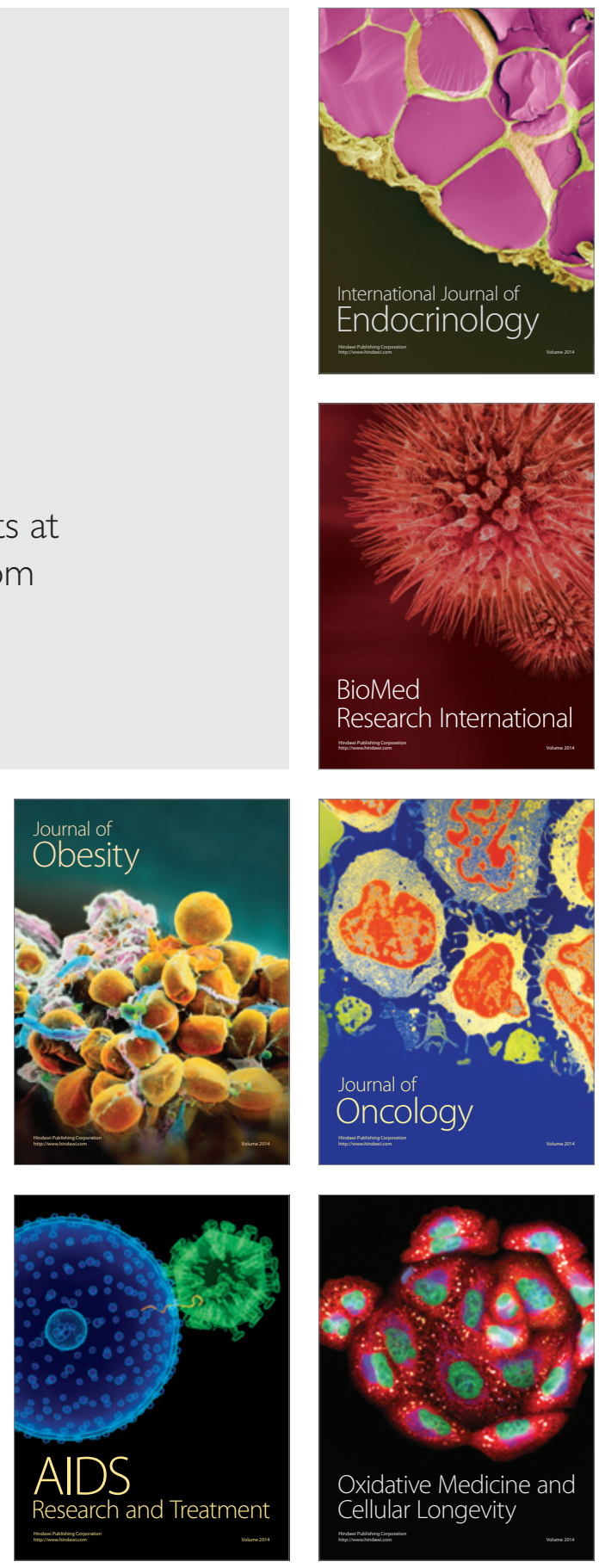